\title{
POTENCIAL MUTAGÊNICO E GENOTÓXICO DE PEIXES EXPOSTOS AO SEDIMENTO DO RIO DOCE APÓS O ROMPIMENTO DA BARRAGEM
}

\author{
Isabela Breder Vargas ${ }^{1}$ \\ Vivian França Mainardi ${ }^{2}$ \\ Luara Louzada Aguiar ${ }^{3}$ \\ Diego Lacerda de Souza ${ }^{4}$ \\ Allan Emilio Piedade ${ }^{5}$ \\ Thiago Pessanha Rangel ${ }^{6}$ \\ Diogo Quitete Ribeiro de Almeida ${ }^{7}$ \\ Pedro Pierro Mendonça ${ }^{8}$ \\ Carlos Eduardo de Rezende ${ }^{9}$ \\ José Augusto de Oliveira David ${ }^{10}$ \\ Cristiane dos Santos Vergilio ${ }^{11}$
}

\begin{abstract}
Resumo: O rompimento da barragem de Fundão em Bento Rodrigues culminou no lançamento de rejeito de minério ao longo do Rio Doce. A caracterização do rejeito liberado e seus prováveis efeitos sobre organismos são importantes no estudo do impacto sobre as populações que foram afetadas. Neste sentido, o presente estudo avaliou o potencial mutagênico dos sedimentos de fundo do leito principal do Rio Doce após o rompimento da barragem contendo rejeito de minério, através da análise do teste de micronúcleo e de outras anormalidades nucleares em tilápias. Os peixes expostos por 7 dias aos sedimentos de diferentes localidades ao longo do Rio Doce apresentaram índices de anormalidades nucleares e micronúcleos, indicando que há a presença de agentes mutagênicos e genotóxicos que podem vir a causar efeitos adversos nos organismos expostos. Esses resultados iniciais levantam a necessidade de estudos sistemáticos e de um monitoramento de longo prazo na região.
\end{abstract}

Palavras-chave: Ecotoxicologia; Eritrócito; Mutagenicidade; Oreochromis niloticus.

\footnotetext{
1 Universidade Federal do Espírito Santo, Brasil. E-mail: isabelabvargas@gmail.com.

2 Universidade Federal do Espírito Santo, Brasil. E-mail: vfmainardi@gmail.com.

3 Universidade Federal do Espírito Santo, Brasil. E-mail: luaralouzada@hotmail.com.

${ }^{4}$ Universidade Estadual do Norte Fluminense Darcy Ribeiro, Brasil. E-mail: lacerdadesouza@hotmail.com.br.

5 Instituto Federal de Educação, Ciência e Tecnologia do Espírito Santo, Brasil. E-mail: allanemilio1996@gmail.com.

6 Universidade Estadual do Norte Fluminense Darcy Ribeiro, Brasil. E-mail: thiprangel@yahoo.com.br.

7 Universidade Estadual do Norte Fluminense Darcy Ribeiro, Brasil. E-mail: diogoquitete@hotmail.com.

8 Instituto Federal de Educação, Ciência e Tecnologia do Espírito Santo, Brasil. E-mail: ppierrom@gmail.com.

9 Universidade Estadual do Norte Fluminense Darcy Ribeiro, Brasil. E-mail: crezendeuenf@yahoo.com.br.

10 Universidade Federal de São Carlos, Brasil. E-mail: joseaugustodavid@hotmail.com.

11 Universidade Federal do Espírito Santo, Brasil. E-mail: cristianesvergilio@gmail.com.
} 